\title{
Machine-learning based Hybrid Method for Surface Defect Detection and Categorization in PU Foam
}

\author{
Shailendra Singh Kathait \\ Co-founder \& Head of Analytics \\ Valiance Solutions Pvt. Ltd. \\ Noida, Uttar Pradesh
}

\author{
Sakshi Mathur \\ Indian Institute of Technology, Roorkee \\ Roorkee, Uttarakhand
}

\begin{abstract}
Foam making is an important industry, their main applications being foam mattresses. Hence, their production in the industries is subject to very strict safety checks to ensure their quality. There are many types of defects that can arise during their manufacturing process, like holes, cuts, a misconfiguration in the material and many more. These defects are reviewed manually which leads to an inadequate accuracy and many defects are not detected. This paper proposes a novel approach that identifies defects in the foam material and on the surface using a hybrid method. Both supervised and unsupervised approaches are used to categorize materials based on normal or defective, including the type of defect. Then the reliable model is chosen according to the precision rates of both the models.
\end{abstract}

\section{Keywords}

Polymerization, Data Augmentation, Computer vision, OpenCV, Image Processing, Machine learning, Deep Learning, Convolutional Neural Networks

\section{INTRODUCTION}

Polyurethane foam is a chemically complex polymeric product having a broad range of load-bearing capability and resiliency. It is used as cushioning material for furniture, bedding, carpeting, packaging and automotive applications. It is best known for supporting our body for a large part of the day, in mattresses, upholstered furniture, and car seats. What is sometimes less known is that we enjoy the benefits of hundreds of polyurethane foam articles without even noticing. [2]

Its applications are virtually endless, ranging from small but essential items such as sponges in the kitchen, medical dressings to large filters and soundproofing systems that keep our environment clean and quiet. Polyurethane is a leading member of the wide-ranging and highly diverse family of polymers or plastics. Polyurethane can be solid or can have an open cellular structure, in which case it is called foam and foams can be flexible or rigid.

A simple explanation, manufacturers make polyurethane foam by reacting polyols and diisocyanates, both products derived from crude oil (Figure 1). A series of additives are necessary to produce high-quality PU foam products, depending on the application the foam will be used for.

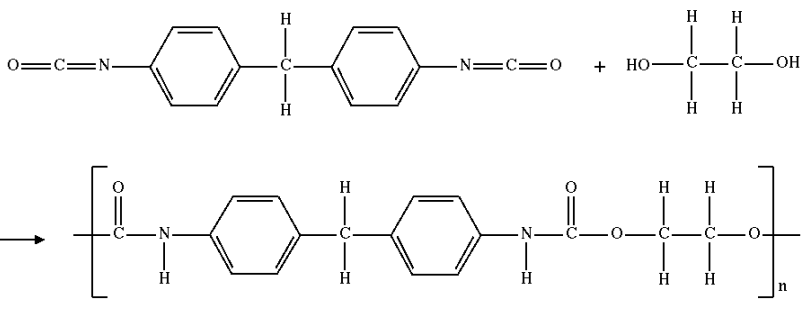

Fig. 1: Polyurethane synthesis, wherein the urethane groups $\mathrm{NH}(\mathrm{C}=\mathrm{O}) \mathrm{O}$ link the molecular units. [16

There are several defects that may appear in the manufacturing of polyfoams in the industries, like improper cross-linking in the polymer, surface defects during cutting or rolling, etc. This calls for a lot of intensive labor work to manually check each and every foam piece after it is made, which of course causes many defects to pass on without being noticed by the worker on duty. This costs losses to the industry as a lot of foam is then wasted because of such defects as this faulty foam will make a low-quality mattress and this is a complete waste. This research is an attempt to reduce the amount of manual work done by the workers and to prevent foam making industries from incurring losses. [14]

This research used two different approaches to solve the problem and determine surface defects in the polyfoam. It focused on a few major visible defects. In particular,on holes, (see-through or partial), cuts in the foam sample, and misconfiguration resulting from density variation in the sample. The codes were tested in labs by taking samples of different types from the industry. The end process of this research is to deploy the code and use it at an industrial level. To deploy the code, Raspberry pi3 board [8] was used which is a credit-card sized electronic board and can run these codes at an ease. To raise an alarm and inform the workers that a defect has been noticed and something needs to be done, an alarm buzzer and a LED light were used. These were connected to a GPIO PCB board which was connected to the raspberry pi 3 board. To make the detection real-time, two high-resolution CCD cameras were used at an angle which would cover the whole surface area of the board under inspection. These cameras were again connected to the pi board. 


\section{PROPOSED METHODOLOGY}

This research work has proposed two new system for efficient defect detection using computer vision and image processing techniques, which are coupled with the pattern discovery algorithms such as predicting the color of the foam material. The new detection system is developed using two different novel approaches:

(1) Computer vision and Image Analysis

(2) Supervised training and categorization

\subsection{Data Acquisition and processing}

This is the first and foremost aspect of an algorithm. For testing the code in laboratory, different images of foam samples were taken at different angles and light conditions to train the model correctly. Foam samples were illuminated from the backside using a source of visible light.

\subsection{Data Cleaning}

Before an image could be used to predict something, it has to be cleaned. Data cleaning is a very important pre-processing step in machine learning. Original data will have many discrepancies that need to be removed, like having outliers, having null data etc. Data discrepancy varies for different types of data an thus there is no defined rule how to clean data and prepare it for machine learning models. [5]

The raw image would give inappropriate and undesired results because of the presence of noise and other factors. To reduce the noise in the captured images, high-resolution cameras were used. The images taken were in RGB images and there was no need of colors to determine the category of foam sample, so, they were converted into grayscale and normalized between 0 to 255 to remove outliers if any (Figure 2). The images were manually labeled according to its category to train the classifier.
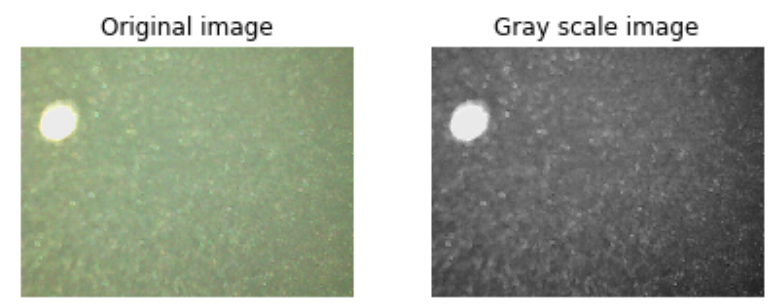

Fig. 2: Data cleaning example

\subsection{Data Augmentation}

Since the amount of data needed to train any model and to get good accuracy is very large, data augmentation was done on the sample data from the foam making industry. It is a way to reduce overfitting in the model and to increase the accuracy.

Data augmentation can be applied to any form of data but may be especially useful for customer data, sales patterns, product sales, where additional information can help provide more in-depth insight. In terms of images, it may mean that increasing the number of images in the dataset. In terms of traditional row/column format data, it means increasing the number of rows or objects.[15]
Some of the augmentation techniques that were used in this research are: (Figure 3)

-Flip: Flipping images horizontally and vertically.

-Rotation: Rotating the image by finer angles.

- Scale: The image can be scaled outward or inward.

-Blur: Blurring the image slightly will enhance the learning capability.

- Gaussian Noise: Adding just the right amount of noise can enhance the learning capability.

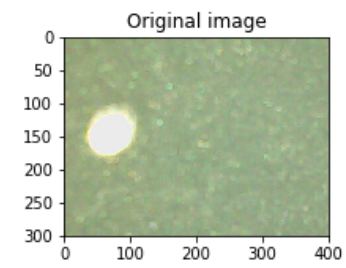

(a) Original Image

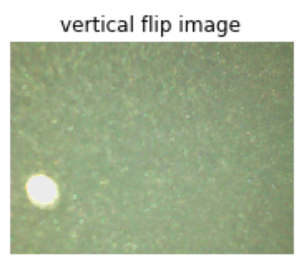

(b) Vertical Flip

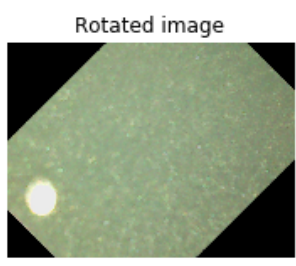

(d) Rotation

blurred image

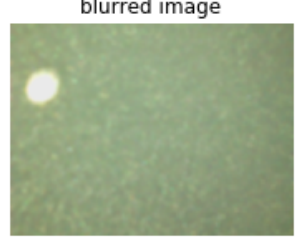

(f) Blurring

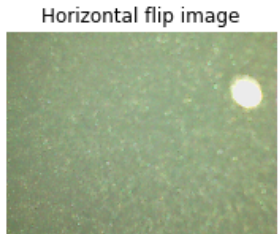

(c) Horizontal Flip

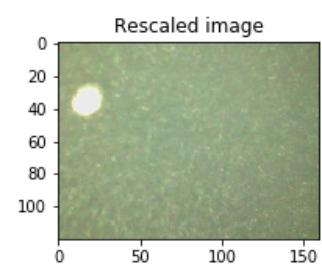

(e) Rescaling

Random noise image

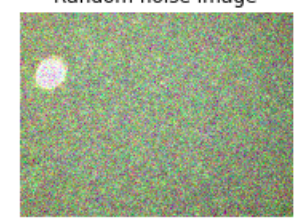

(g) Gaussian Noise

Fig. 3: Data augmentation techniques on original image data 


\section{COMPUTER VISION AND IMAGE ANALYSIS}

Computer vision is a field of computer science that works on enabling computers to see, identify and process images in the same way that human vision does, and then provide the appropriate output. It is like imparting human intelligence and instincts to a computer. The human eye is a complex structure and it goes through a more complex phenomenon of understanding the environment. In a similar fashion, making machines see things and make them capable enough to figure out what they are seeing and further categorize it, is still a pretty tough job.[12]

The goal of Computer Vision is to emulate human vision using digital images through three main processing components, executed one after the other:

(1) Image acquisition: Image acquisition is the process of translating the analog world around us into binary data composed of zeros and ones, interpreted as digital images.

(2) Image processing: The second component of Computer Vision is the low-level processing of images. Algorithms are applied to the binary data acquired in the first step to infer low-level information on parts of the image. This type of information is characterized by image edges, point features or segments, for example. They are all the basic geometric elements that build objects in images.

(3) Image analysis and understanding: The last step of the Computer Vision pipeline if the actual analysis of the data, which will allow the decision making. High-level algorithms are applied, using both the image data and the low-level information computed in previous steps.

As our human visual understanding of the world is reflected in our ability to make decisions through what we see, providing such a visual understanding of computers would allow them the same power.

\subsection{Digital Image Processing}

Image processing is a method to perform some operations on an image, in order to get an enhanced image or to extract some useful information from it. It is a type of signal processing in which input is an image and output may be image or characteristics/features associated with that image. Nowadays, image processing is among rapidly growing technologies. It forms core research area within engineering and computer science disciplines too. [6]

Image processing basically includes the following three steps:

-Importing the image via image acquisition tools;

—Analyzing and manipulating the image;

-Output in which result can be altered image or report that is based on image analysis.

The three general phases that all types of data have to undergo while using digital technique are pre-processing, enhancement, and display, information extraction.

\subsection{OpenCv}

OpenCV (Open Source Computer Vision Library) is an open source computer vision and machine learning software library. OpenCV was built to provide a common infrastructure for computer vision applications and to accelerate the use of machine perception in the commercial products. The library has more than 2500 optimized algorithms, which includes a comprehensive set of both classic and state-of-the-art computer vision and machine learning algorithms.
These algorithms can be used to detect and recognize faces, identify objects, classify human actions in videos, track camera movements, track moving objects, extract 3D models of objects, produce 3D point clouds from stereo cameras, stitch images together to produce a high resolution image of an entire scene, find similar images from an image database, remove red eyes from images taken using flash, follow eye movements, recognize scenery and establish markers to overlay it with augmented reality, etc. [9]

\subsection{Approach}

The surface chemistry of polyurethane foam leads to it passing a few amounts of visible light illuminated on it through a light source. Also, if there is a cut or a density gradient in the surface of the foam material, this will cause a difference in light received from the foam sample. A defect area will naturally be more illuminated as compared to rest of the non-defective areas of the sample. We used this property of polyfoams to build a classifier which will tell whether the foam sample is defective or normal. This was an unsupervised approach of detection using computer vision techniques and thus, the defect could not be categorized into its different types.

The algorithm took an image of foam and did pre-process on it like removing noise and converting it to grayscale from RGB color code. Then Gaussian blur filter was applied on the grayed image to smoothen out the edges and remove noise from the image. A series of morphological dilation and erosion were then applied on the image.[1] Dilation adds pixels to the boundaries of objects in an image, while erosion removes pixels on object boundaries. The number of pixels added or removed from the objects in an image depends on the size and shape of the structuring element used to process the image. These processes will lead to a clear distinction that a computer can visualize because of thresholding. The computer will then count the number of defects in the image using masking. If it is greater than 0 , it means the given sample has a defect in it which must be checked. To detect the location of the fault, object localization technique was used. This will create a bounding circle at the given coordinates surrounding the defect which can be viewed on the screen to calculate the accuracy of our algorithm.

\subsection{Evaluation and Validation}

The algorithm was tested for a testing data comprising 40 illuminated samples' images. The output was similar to figure 4 .

To evaluate the model, a confusion matrix was made. Here, faulty is considered as 1 and normal as 0 . The matrix was something like this:

$\begin{array}{ccc} & 1 & 0 \\ 1 & 20 & 8 \\ 0 & 2 & 10\end{array}$

Precision $=\frac{T P}{T P+F P}$

Precision $=\frac{30}{30+5}=0.857$

Recall $=\frac{T P}{T P+F N}$

Recall $=\frac{3}{30+1}=0.967$

F Score $=2 * \frac{\text { Recall } * \text { Precision }}{\text { Recall }+ \text { Precision }}$

F Score $=2 * \frac{0.857 * 0.967}{0.857+0.967}=0.928$ 


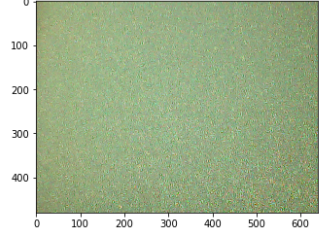

(a) Normal

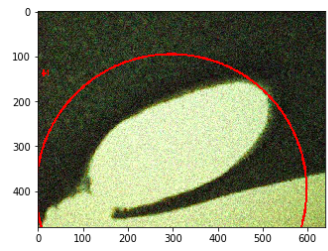

(c) $\mathrm{Cut}$

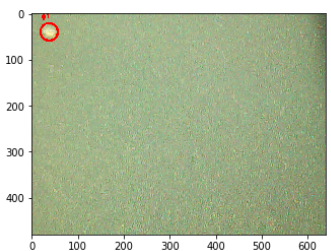

(b) Hole

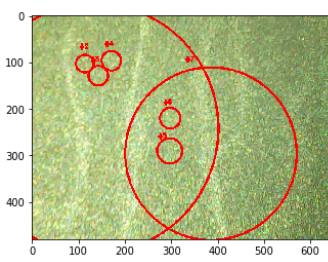

(d) Misconfiguration
Fig. 4: Types of different test predictions

\section{SUPERVISED TRAINING AND CATEGORIZATION}

Machine learning is a core sub-area of artificial intelligence; it enables computers to get into a mode of self-learning without being explicitly programmed. When exposed to new data, these computer programs are enabled to learn, grow, change, and develop by themselves. It allows computers to find insightful information without being programmed where to look for a particular piece of information; instead, it does this by using algorithms that iteratively learn from data. With the constant evolution of the field, there has been a subsequent rise in the uses, demands, and importance of machine learning. Big data has become quite a buzzword in the last few years; thats in part due to increased sophistication of machine learning, which helps analyze those big chunks of big data. Machine learning has also changed the way data extraction, and interpretation is done by involving automatic sets of generic methods that have replaced traditional statistical techniques.[11]

The process of learning begins with observations or data, such as examples, direct experience, or instruction, in order to look for patterns in data and make better decisions in the future based on the examples that we provide. The primary aim is to allow the computers to learn automatically without human intervention or assistance and adjust actions accordingly.

Machine learning algorithms are often categorized as supervised or unsupervised.[17]

(1) Supervised machine learning algorithms can apply what has been learned in the past to new data using labeled examples to predict future events. Starting from the analysis of a known training dataset, the learning algorithm produces an inferred function to make predictions about the output values. The system is able to provide targets for any new input after sufficient training. The learning algorithm can also compare its output with the correct, intended output and find errors in order to modify the model accordingly.

(2) In contrast, unsupervised machine learning algorithms are used when the information used to train is neither classified nor labeled. Unsupervised learning studies how systems can infer a function to describe a hidden structure from unlabeled data. The system doesnt figure out the right output, but it explores the data and can draw inferences from datasets to describe hidden structures from unlabeled data.

\subsection{Deep Learning}

Deep Learning, a prominent topic in Artificial Intelligence domain, has been in the spotlight for quite some time now. While traditional machine learning algorithms are linear, deep learning algorithms are stacked in a hierarchy of increasing complexity and abstraction. Each algorithm in the hierarchy applies a nonlinear transformation on its input and uses what it learns to create a statistical model as output. Iterations continue until the output has reached an acceptable level of accuracy. The number of processing layers through which data must pass is what inspired the label deep.[10]

\subsection{Convolutional Neural Networks}

A convolutional neural network (CNN, or ConvNet) is a class of deep, feed-forward artificial neural networks, most commonly applied to analyzing visual imagery. CNN, like neural networks, are made up of neurons with learnable weights and biases. Each neuron receives several inputs, takes a weighted sum over them, pass it through an activation function and responds with an output. The whole network has a loss function. ConvNet architectures make the explicit assumption that the inputs are images.[13]

\subsection{Approach}

In order to make the foam defect detection system, first a dataset was made by collecting images from the foam manufacturing factory. The experiments were focused on three different surface defects:

1) Holes

2) Cuts

3) Misconfiguration

To construct the dataset, 3000 images were analyzed using foam samples and applying data augmentation on those images to multiply them using effects like rotation, Gaussian blurring, adding or removing noise etc. In particular, the dataset used 1500 normal images, and 500 of each defect to create a balanced training data. Then the dataset was split into training and validation data in the ratio of 4:1. A testing dataset was made of 40 images to evaluate the performance of the model in the end. Four categories were made to classify the sample image as "normal", hole, cuts or misconfiguration. Then a sequential model was trained on a fully connected neural network [18]. The output was categorized as $[0,1,2,3]$ indicating the different categories mentioned above respectively. The model consisted of 1 input layer and 4 conv layers of filter size $32,32,32,64$ and 64 respectively. The input was kept to be of size $(100,100,1)$. ReLU (Rectified Linear Unit) activation function was used for these layers, which may be defined as:

$f(x)=x^{+}=\max (0, x)[3$

Also, 3 pooling layers and dropout layers were added with these conv layers. Maxpool of size $(2,2)$ was used. Then added a fully connected layer and an output layer in the last. The output layer consisted of softmax activation function. In mathematics, the softmax function, or normalized exponential function, is a generalization of the logistic function that "squashes" a K-dimensional vector $\mathrm{z}$ of arbitrary real values to a K-dimensional vector $\sigma(z)$ of real val- 
ues, where each entry is in the range $(0,1]$, and all the entries add up to 1 . The function is given by:

$$
\begin{aligned}
& \sigma: R^{K} \rightarrow\left\{\sigma \in R^{K} \mid \sigma_{i}>0, \sum_{i=1}^{K} \sigma_{i}=1\right. \\
& \sigma(\mathbf{z})_{j}=\frac{e^{z_{j}}}{\sum_{k=1}^{K} e^{z_{k}}} \text { for } j=1,, K .
\end{aligned}
$$

The model was compiled using an optimizer and was trained for a batch size of 86 images with total 50,000 epochs. This model was trained on a 64GB RAM computer for faster training and then the architecture and weights were saved to be loaded on any other device and testing.

\subsection{Evaluation and Validation}

The model gave a training accuracy of $95.18 \%$ while a validation accuracy of $97.70 \%$. It was tested on the testing data which comprised 40 random images. The results were similar to figure 5 . Here 0 stands for normal images, 1 for holes, 2 for cuts and 3 for misconfiguration.
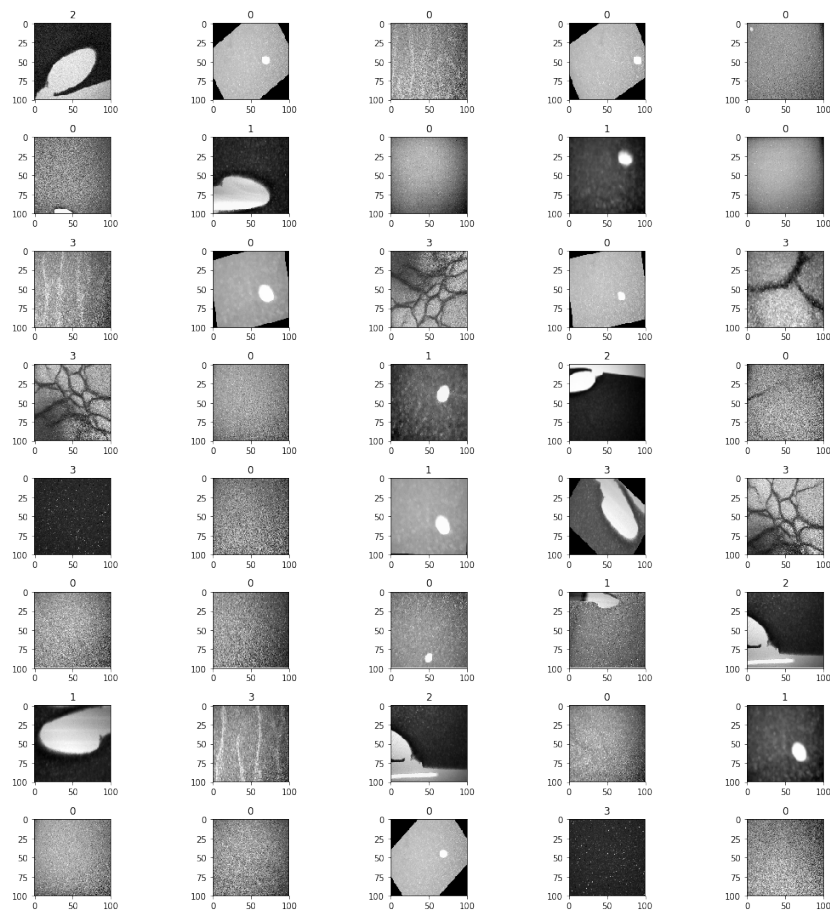

Fig. 5: Test predictions

To evaluate the model, again a confusion matrix was made. Here, faulty is considered as 1 and normal as 0 . The matrix was something like this:

$\begin{array}{ccc} & 1 & 0 \\ 1 & 20 & 8 \\ 0 & 2 & 10\end{array}$

Precision $=\frac{T P}{T P+F P}[7$

Precision $=\frac{20}{20+2}=0.909$

Recall $=\frac{T P}{T P+F N}$

Recall $=\frac{20}{20+8}=0.714$

F Score $=2 * \frac{\text { Recall } * \text { Precision }}{\text { Recall }+ \text { Precision }}$

F Score $=2 * \frac{0.714 * 0.909}{0.714+0.909}=0.78$

\section{RESULTS}

The F Score of both the approaches was quite promising and gave hope that these can be used as an industrial level to reduce manual work. The computer vision approach is an unsupervised approach and does not need a starting database. Thus it could be deployed first on the raspberry pi3 board. All the images were uploaded with their tags of being normal or defected on the Amazon S3 bucket. After running these for a couple of months, enough data was collected to train the neural networks classifier. The architecture and weights of the CNN model could be loaded on the raspberry pi3 board to run a real-time supervised detector.

\section{CONCLUSION AND FUTURE WORK}

Polyurethane foam, or foam in general, is an important sector of the industry. The uses of foams made by these industries are endless and thus there is a need to ensure the quality of foams produced. Till now these industries use manual work to detect faults and defects included in the foam product which leads to low accuracy, higher labor work and thus overall loss to the industry.

In this paper, two novel approaches are proposed based on computer vision and machine learning in order to detect and categorize surface defects on polyurethane foam during their manufacturing in the industries. This approach starts by retrieving sample images from the industry unit, then applying the two algorithms on these images, and finally to deploy the code to make it a real-time detection system which can be installed in the industries.

The future work in this approach can be to increase the accuracy of detection, include more classes of faults and detect very minor defects that may be even hard to visualize manually also. One thing where the algorithm faced issue was detection in dim lights, which we are finding a solution for.

\section{REFERENCES}

[1] Morphological Processing, chapter 8, pages 197-234. WileyBlackwell, 2011.

[2] John Akindoyo, Md Dalour Hossen Beg, Suriati Ghazali, Muhammad Islam, Nitthiyah Jeyaratnam, and Yuvaraj Ar. Polyurethane types, synthesis and applications-a review. 6:114453-114482, 112016.

[3] Raman Arora, Amitabh Basu, Poorya Mianjy, and Anirbit Mukherjee. Understanding deep neural networks with rectified linear units. In International Conference on Learning Representations, 2018.

[4] Dunne Campbell, R. A. Dunne, and N. A. Campbell. On the pairing of the softmax activation and cross-entropy penalty functions and the derivation of the softmax activation function. 
[5] Xu Chu, Ihab F. Ilyas, Sanjay Krishnan, and Jiannan Wang. Data cleaning: Overview and emerging challenges. In Proceedings of the 2016 International Conference on Management of Data, SIGMOD '16, pages 2201-2206, New York, NY, USA, 2016. ACM.

[6] Rafael C. Gonzalez and Richard E. Woods. Digital image processing. Prentice Hall, Upper Saddle River, N.J., 2008.

[7] Cyril Goutte and Eric Gaussier. A probabilistic interpretation of precision, recall and f-score, with implication for evaluation. In Proceedings of the 27th European Conference on Advances in Information Retrieval Research, ECIR'05, pages 345-359, Berlin, Heidelberg, 2005. Springer-Verlag.

[8] Gareth Halfacree and Eben Upton. Raspberry Pi User Guide. Wiley Publishing, 1st edition, 2012.

[9] Itseez. The OpenCV Reference Manual, 2.4.9.0 edition, April 2014.

[10] Alex Krizhevsky, Ilya Sutskever, and Geoffrey E Hinton. Imagenet classification with deep convolutional neural networks. In F. Pereira, C. J. C. Burges, L. Bottou, and K. Q. Weinberger, editors, Advances in Neural Information Processing Systems 25, pages 1097-1105. Curran Associates, Inc., 2012.

[11] Thomas M. Mitchell. Machine Learning. McGraw-Hill, Inc., New York, NY, USA, 1 edition, 1997.

[12] Sebastian Nowozin and Christoph H. Lampert. Structured learning and prediction in computer vision. Found. Trends. Comput. Graph. Vis., 6(3\&\#8211;4):185-365, March 2011.

[13] Keiron O'Shea and Ryan Nash. An introduction to convolutional neural networks. 112015.

[14] Iker Pastor-Lopez, Igor Santos, Aitor Santamaria-Ibirika, Mikel Salazar, Jorge de La-PeA-Sordo, and Pablo Bringas. Machine-learning-based surface defect detection and categorisation in high-precision foundry, 072012.

[15] Luis Perez and Jason Wang. The effectiveness of data augmentation in image classification using deep learning. CoRR, abs/1712.04621, 2017.

[16] Laura Rducu, Cristina Cozma, A.E.B. Stroescu, Adelaida Avino, M.D. Tanasescu, D.G. Balan, and Cristian Jecan. Our experience in chronic wounds care with polyurethane foam. 69:585-586, 032018.

[17] R. Sathya. Comparison of supervised and unsupervised learning algorithms for pattern classification.

[18] Matthew D. Zeiler and Rob Fergus. Visualizing and understanding convolutional networks. CoRR, abs/1311.2901, 2013. 